ORIGINAL ARTICLE

\title{
Chorioamnionitis increases matrix metalloproteinase-8 concentrations in bronchoalveolar lavage fluid from preterm babies
}

\author{
A E Curley, D G Sweet, K J MacMahon, C M O'Connor, H L Halliday
}

Arch Dis Child Fetal Neonatal Ed 2004;89:F61-F64

See end of article for authors' affiliations

\section{Correspondence to:} Dr Sweet, Grosvenor Road, Belfast BT12 6BB, Northern Ireland: dsweet@dnet.co.uk

Accepted 21 January 2003

\begin{abstract}
Objective: To determine the effects of chorioamnionitis and antenatal corticosteroids on matrix metalloproteinase-8 (MMP-8) concentrations in bronchoalveolar lavage (BAL) fluid from preterm babies in the first week of life.

Design: Prospective observational study.

Setting: Regional neonatal intensive care unit.

Patients: Thirty five ventilated preterm babies $<33$ weeks gestation, seven of whom were born after chorioamnionitis, which was diagnosed histologically as the presence of inflammatory cells in the chorioamnionic plate.

Methods: MMP-8 was measured by enzyme linked immunosorbent assay (ELISA) in 90 serial BAL samples taken during the first six postnatal days. The median MMP-8 concentration for each baby was calculated. Results: Median MMP-8 concentrations were higher in the chorioamnionitis group than in those without $(43 \vee 5 \mathrm{ng} / \mathrm{ml})$. Partial or complete courses of antenatal corticosteroids had no effect on MMP-8 concentrations.

Conclusions: Higher concentrations of MMP-8 are found in BAL fluid from preterm babies from pregnancies complicated by chorioamnionitis. This type I collagenase may contribute to the lung injury that occurs in some babies with respiratory distress syndrome.
\end{abstract}

horioamnionitis is a major risk factor for preterm delivery and adverse neonatal outcome. ${ }^{12}$ The immune response to chorioamnionitis involves the recruitment of polymorphonuclear leucocytes into the amniotic cavity. ${ }^{3}$ Secretory products of these neutrophils are increased in cases of chorioamnionitis, preterm rupture of membranes, and preterm delivery. ${ }^{4}$ Increased concentrations of one secretory product of neutrophils, matrix metalloproteinase-8 (MMP8 ), in amniotic fluid is associated with adverse neonatal outcome including chronic lung disease (CLD) after preterm delivery. ${ }^{6}$ MMP-8 is also known as neutrophil collagenase and it predominantly cleaves type-I collagen, which is a major structural protein of the lung extracellular matrix. ${ }^{7}$ It is released from intracellular granules mainly during inflammatory neutrophil activation and may have a role in neutrophil adhesion and transendothelial migration. ${ }^{8}$ Its activity is normally regulated by inhibitors called tissue inhibitor of metalloproteinases. ${ }^{9}$ An imbalance between MMP-8 and these inhibitors may lead to excessive proteolysis of the lung extracellular matrix during inflammatory lung injury.

Amniotic fluid neutrophils are thought to be of fetal rather than maternal origin. ${ }^{10}$ If their presence in amniotic fluid represents a fetal systemic inflammatory response, then it is possible that this persists into the early postnatal period. ${ }^{11}$ Continued release of neutrophil secretory products such as MMP-8 could potentially lead to increased degradation of the collagenous content of the extracellular matrix of the neonatal lung. We have previously shown an association between elevated MMP-8 concentrations in preterm neonatal bronchoalveolar lavage (BAL) fluid and the subsequent development of CLD. ${ }^{12}$ We have hypothesised that the increased risk of CLD associated with chorioamnionitis ${ }^{13}$ may be related to excess release of neutrophil collagenase (MMP-8) which can disrupt the lung extracellular matrix.
The objective of this study was to determine whether or not MMP-8 concentrations in BAL fluid from preterm babies within the first week of life are increased after pregnancies complicated by chorioamnionitis. We also wanted to determine the effect of antenatal betamethasone on concentrations of MMP-8 in BAL fluid.

\section{METHODS}

\section{Patients}

This was a prospective study of babies born in the Royal Maternity Hospital, Belfast. The research ethics committee of Queen's University Belfast gave approval for the study, and parental consent was obtained before babies were enrolled. Thirty five babies of less than 33 weeks gestation were recruited when they required intubation and mechanical ventilation in the first 2 days of life, and 90 samples of BAL fluid were obtained. Outcomes for about one third of these babies have already been reported..$^{12}$

\section{Bronchoalveolar lavage}

Serial BAL was performed daily whenever possible from time of intubation until extubation or the 6th day of life, whichever was soonest. Babies were only studied when clinically stable and not during the $12 \mathrm{~h}$ after a dose of surfactant. BAL was performed in a standardised way using a well established technique. ${ }^{14}$ No marker of dilution was used, in accordance with the most recent European Respiratory Society guidelines on BAL. ${ }^{15}$ In summary, $1 \mathrm{ml} / \mathrm{kg}$ sterile $0.9 \%$ saline was instilled using a $2 \mathrm{ml}$ syringe through a $5 \mathrm{~F}$ gauge feeding catheter which had been placed using the endo-

Abbreviations: $B A L$, bronchoalveolar lavage; $C L D$, chronic lung disease; IQR, interquartile range; MMP-8, matrix metalloproteinase-8 
tracheal tube in the distal right main bronchus. The saline was instilled and immediately aspirated back into the syringe. The median volume returned was $0.7 \mathrm{ml}$ (interquartile range (IQR) $0.5-1.2 \mathrm{ml}$ ). A sample was considered technically satisfactory if more than $40 \%$ of the instilled volume was returned. The sample was clarified by centrifugation at $1500 \mathrm{~g}$ for five minutes at room temperature, and the supernatant immediately frozen at $-70^{\circ} \mathrm{C}$ for subsequent analysis.

\section{Definitions}

Gestation was estimated by duration of amenorrhoea combined with early ultrasound assessment. Chorioamnionitis was defined histologically as the presence of inflammatory cells in the chorioamnionic plate. A consensus on each diagnosis was reached by two consultant pathologists (Dr Denis O' Hara and Dr Claire Thornton, Department of Pathology, Royal Victoria Hospital, Belfast) who examined all placentas in this study. A complete course of antenatal corticosteroids was defined as two doses of $12 \mathrm{mg}$ betamethasone given intramuscularly 12-24 h apart and followed by a $24 \mathrm{~h}$ period before delivery. The severity of the initial respiratory disease was assessed using the median arterial-alveolar oxygen tension ratio $\left(\mathrm{a} / \mathrm{A}^{\mathrm{P}} \mathrm{O}_{2}\right)$ in the first 24 hours of life. This was calculated from arterial blood gases using the formula:

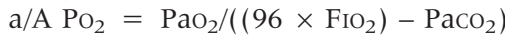

where $\mathrm{PaO}_{2}$ and $\mathrm{PaCO}_{2}$ are expressed in $\mathrm{kPa}$, and $\mathrm{FIO}_{2}$ as a fraction of 1 . CLD was defined as the requirement for supplemental oxygen to maintain oxygen saturation measured by pulse oximetry greater than $92 \%$ at 36 weeks postconceptional age.

\section{Assay of MMP-8}

Samples were assayed for MMP-8 by enzyme linked immunosorbent assay (ELISA; Amersham International, Amersham, Bucks, UK). MMP-8 concentration was determined by a three step system. Briefly, $100 \mu \mathrm{l}$ samples of BAL fluid were added to wells of a microtitre plate coated with a solid phase monoclonal antibody to MMP-8. After incubation at $25^{\circ} \mathrm{C}$ for two hours, plates were washed four times in $0.01 \mathrm{M}$ phosphate buffer ( $\mathrm{pH} 7.5$ containing $0.05 \%$ Tween $20)$. Then $100 \mu \mathrm{l}$ of a rabbit polyclonal antibody to MMP-8 was added and incubated at $25^{\circ} \mathrm{C}$ for a further two hours. Antibody bound to MMP-8 in the sample was then detected by incubation with horseradish peroxidase-conjugated donkey anti-rabbit $\operatorname{IgG}\left(25^{\circ} \mathrm{C}\right.$, one hour $)$ followed by addition of horseradish peroxidase substrate, $3^{\prime}, 3^{\prime}, 5^{\prime}, 5^{\prime}$-tetramethylbenzidine/hydrogen peroxide in $20 \%$ dimethyl formamide. After incubation at $25^{\circ} \mathrm{C}$ for 30 minutes, enzyme activity was quenched by the addition of $100 \mu \mathrm{l} \mathrm{l} \mathrm{M}$ sulphuric acid, and absorbance read at $450 \mathrm{~nm}$ on a microtitre plate reader (Ultraspec plus; Pharmacia, Cambridge, UK). The sensitivity of the MMP- 8 assay was $0.032 \mathrm{ng} / \mathrm{ml}$ (range $0.032-4 \mathrm{ng} / \mathrm{ml}$ ). The ability of the assay to detect MMP- 8 in dilute biological fluids was confirmed by assessment of concentrations in supernatants from phorbol myristrate acetate stimulated alveolar macrophages. The ELISA system we used measured total concentrations of latent and active forms of MMP-8 and any MMP- 8 bound to inhibitor. All samples were assayed in duplicate. Results were confirmed by Western blotting.

\section{Statistical analysis}

The median MMP-8 concentration was calculated using all of the samples from each baby, and this was used as the summary statistic. Median MMP-8 concentrations were not normally distributed, so non-parametric statistical tests were used. However, the logarithm of MMP-8 concentration was normally distributed, and this was used in the logistic regression analyses. Fishers' exact test, Spearman correlation, Mann-Whitney U test, and regression analysis were calculated using SPSS for Windows version 9.0, 1996 as appropriate.

\section{RESULTS}

Ninety BAL samples were collected for analysis. Each baby had on average two BAL samples taken (range one to six). Seven of the 35 babies were from pregnancies complicated by chorioamnionitis. There was no difference in the mean proportion of BAL fluid volume returned between the chorioamnionitis and no chorioamnionitis groups $(60 \% v$ $65 \%$ ). The babies from pregnancies with chorioamnionitis were less mature and lighter at birth than those from the non-chorioamnionitis group (table 1). The initial severity of respiratory distress in the babies in the chorioamnionitis

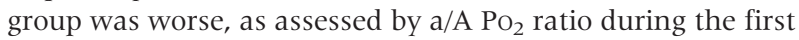
24 hours. However, they were not more likely to develop CLD (table 1).

MMP-8 concentrations did not correlate with postnatal age ( $\mathrm{n}=90, r=0.14 ; \mathrm{p}=0.26$ ) (fig 1). Median MMP-8 concentrations decreased with increasing gestational age ( $\mathrm{n}=35, r=-0.32, \mathrm{p}=0.03)$ and birth weight $(\mathrm{n}=35$, $r=-0.38, \mathrm{p}=0.019$ ) (fig 2).

MMP-8 concentrations in the first 6 days of life were higher in the chorioamnionitis group than the non-chorioamnionitis group (fig 3 ). To ensure that this difference was not a function of the differences in gestation between the two groups, the data were submitted to multiple logistic regression analysis. Log (MMP-8 concentration) remained significantly higher in the chorioamnionitis group after correction for differences in gestation and birth weight $(p=0.026)$. MMP-8 concentrations were also raised in the eight babies with a history of prolonged rupture of membranes compared with the 27 without (median (IQR), $18(8-44) v 5(0-8.5) \mathrm{ng} /$ $\mathrm{ml} ; \mathrm{p}=0.047)$.

Thirty $(86 \%)$ of the infants had received at least one dose of antenatal betamethasone. Only 12 (34\%) had a complete course of antenatal corticosteroids. We compared the concentrations of MMP-8 in infants who had received any antenatal corticosteroids with those who did not. There was no significant difference between the two groups (antenatal corticosteroids, $5(2-13) \mathrm{ng} / \mathrm{ml}$; no antenatal corticosteroids, $6(0-54) \mathrm{ng} / \mathrm{ml} ; \mathrm{p}=0.81)$. We also compared infants who had received a complete course of antenatal corticosteroids with those who had received an incomplete course and found no difference in MMP-8 concentrations (complete, 8 (212) $\mathrm{ng} / \mathrm{ml}$; incomplete, $4.5(2-13) \mathrm{ng} / \mathrm{ml} ; \mathrm{p}=0.68$ ) (fig 4$)$.

\begin{tabular}{llll} 
Table 1 & \multicolumn{3}{l}{ Clinical characteristics of the infants recruited } \\
\hline & $\begin{array}{l}\text { Chorioamnionitis } \\
(\mathbf{n}=7)\end{array}$ & $\begin{array}{l}\text { No chorioamnionitis } \\
(\mathbf{n}=\mathbf{2 8})\end{array}$ & $\mathbf{p}$ Value \\
& $740(687-964)$ & $950(730-1501)$ & 0.06 \\
\hline Birth weight (g) & $24.8(24.0-25.7)$ & $26.0(26.0-28.5)$ & 0.016 \\
Gestation (weeks) & $5(71)$ & $3(11)$ & 0.003 \\
PROM & $6(86)$ & $27(86)$ & 1.00 \\
ANCS (any) & $1(14)$ & $14(39)$ & 0.38 \\
ANCS (complete & & $18: 10$ & 0.4 \\
course) & $3: 4$ & $12(42)$ & 1.00 \\
M/F & $0.35(0.24-0.39)$ & $0.40(0.33-0.55)$ & 0.002 \\
a/A PaO 2 ratio & $3(42)$ & & \\
CLD & & &
\end{tabular}

Data shown as median (interquartile rang) or number (\%). PROM, Prolonged rupture of membranes ( $>24$ hours); ANCS, antenatal corticosteroids; a/ $\mathrm{A} \mathrm{PO}_{2}$ ratio, median arterial to alveolar oxygenation index over first 24 hours of life; CLD, chronic lung disease. 


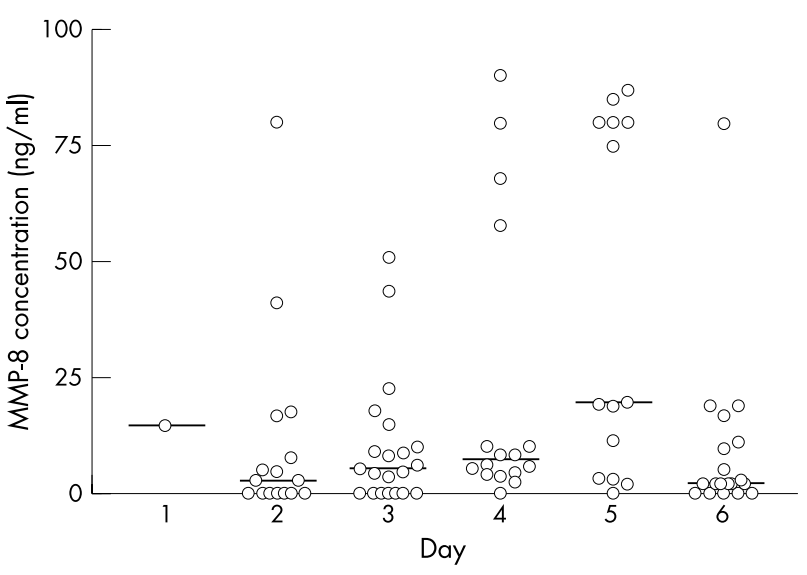

Figure 1 Matrix metalloproteinase-8 (MMP-8) concentrations over the first 6 days of life. Median (IQR) MMP-8 concentrations in $\mathrm{ng} / \mathrm{ml}$ for 48 hour periods from day $1-6$ are shown (days 1-2, 3 (0-15); days 3-4, 6 $(3-11)$; days $5-6,4(2-20) \mathrm{ng} / \mathrm{ml} ; r=0.14 ; \mathrm{p}=0.26)$.

\section{DISCUSSION}

Chorioamnionitis is a risk factor for CLD in the preterm neonate. ${ }^{13}$ Neutrophils that migrate into the fetal and neonatal lung as a consequence of chorioamnionitis may cause damage by protease and oxidant release. There is a correlation between the numbers of neutrophils in the airways of infants with respiratory distress syndrome and the subsequent development of CLD. ${ }^{16}$ MMP-8 concentrations are higher in BAL fluid from babies who develop CLD, ${ }^{12}$ and we hypothesised that a possible mechanism for this may be chorioamnionitis induced fetal lung inflammation.

We found that there was an association between chorioamnionitis and increased BAL fluid concentrations of MMP-8. However, we were surprised that the proportion of babies who developed CLD was no higher in the chorioamnionitis than the non-chorioamnionitis group. This can probably be explained by the small numbers of babies in this study and the fact that postnatal factors such as mechanical ventilation and sepsis have a role in combination with antenatal infection in the pathogenesis of CLD. ${ }^{17}$ More babies in the chorioamnionitis group had prolonged rupture of membranes, and this has been suggested to reduce the risk of respiratory distress syndrome and may reduce CLD, but this remains controversial. ${ }^{18}$ We found that the initial severity of the respiratory disease was higher in the chorioamnionitis group, suggesting that early rupture of membranes or inflammation did not reduce the risk of acute respiratory distress syndrome in our study.

MMP-8 is a $75 \mathrm{kDa}$ enzyme which is predominantly expressed by neutrophils. It is unique among the family of MMPs because of its exclusive expression in inflammatory conditions. ${ }^{19}$ It is upregulated mainly by proinflammatory cytokines, but may also be activated by bacterial proteases through the so called cysteine switch and also through free radical generation. ${ }^{20}{ }^{21}$ It preferentially digests type I collagen, a major matrix protein in the lung interstitium. ${ }^{22}$ It is synthesised during the myelocyte stage of neutrophil development, stored intracellularly within specific granules and released by chemotactic stimulation in vitro and in vivo. ${ }^{23}$ Once released and activated through proteolytic or oxidative mechanisms, the enzyme appears to play a major role in the connective tissue turnover that occurs in inflammatory processes. In addition to digesting connective tissue components, MMP- 8 can also activate $\alpha_{1}$ antitrypsin, and this may be important in the generation of emphysema in adults. ${ }^{24}$

The lung extracellular matrix is required for normal cell alignment and differentiation, and matrix degradation may

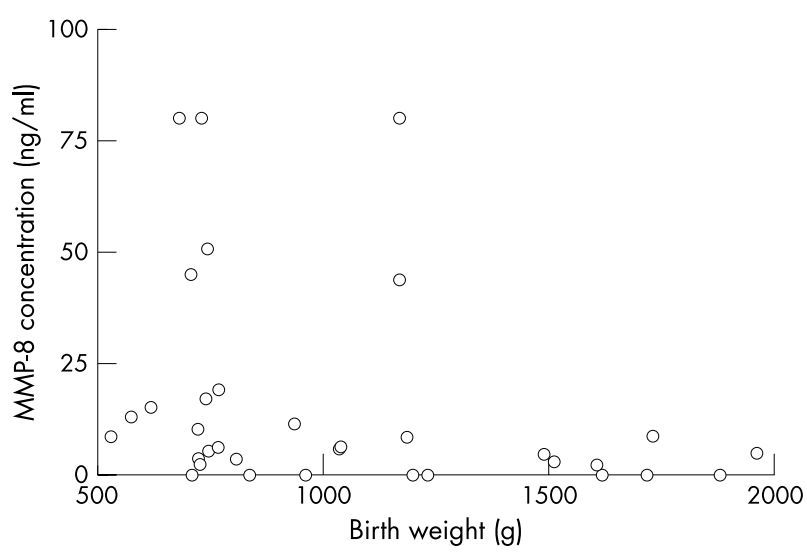

Figure 2 Matrix metalloproteinase-8 (MMP-8) concentrations decreased with increasing birth weight $(n=35, r=-0.38, p=0.019)$.

be what leads to the disordered healing and abnormal lung growth found in babies with CLD. ${ }^{25} 26$

MMP- 8 release and activation by inflammatory mediators suggest that concentrations of this proteinase may be controlled by anti-inflammatory agents such as corticosteroids. Dexamethasone suppresses the endogenous release of interleukin 8 from polymorphonuclear leucocytes from the newborn as well as decreasing associated chemotactic activity. ${ }^{27}$ Treatment with dexamethasone leads to reductions in the breakdown products of type I collagen such as the terminal telopeptide of type I collagen and the C-terminal propeptide of type I collagen. ${ }^{28}$ Corticosteroids also inhibit the production of MMPs by in vitro cultured macrophages. ${ }^{29}$

We did not show any effect of either single or multiple doses of antenatal betamethasone on the concentrations of MMP-8. This was surprising as corticosteroids, by decreasing the migration of neutrophils to the lung, would have been expected to reduce the concentration of MMP-8 or neutrophil collagenase. ${ }^{27}$ The timing of antenatal steroid administration may be relevant. For example, treatment of a mother with steroids five days before delivery may confer benefits to the fetus in terms of lung maturation, but the anti-inflammatory effects may have worn off. Antenatal corticosteroids are known to result in only physiological increases in cord blood steroid concentrations, ${ }^{30}$ and perhaps pharmacological doses are required to produce the desired suppression in neutrophil protease release. There is also a complex interplay between antenatal inflammation, postnatal hypoxia and hyperoxia, and varying degrees of ventilatory barotrauma to which these infants are exposed, which may negate any effects of antiinflammatory mediators.

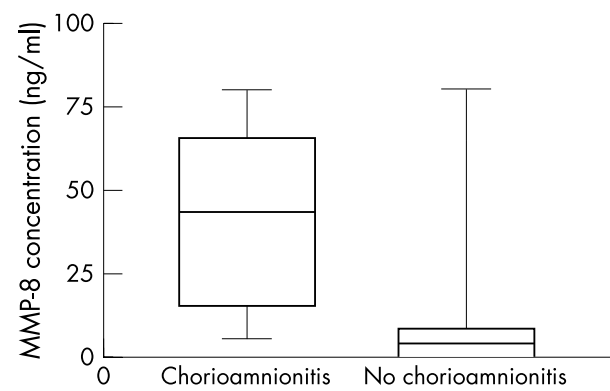

Figure 3 Box and whisker plots depicting median, interquartile range (IQR), and full range of matrix metalloproteinase-8 (MMP-8) concentrations for babies from pregnancies complicated by chorioamnionitis and those without chorioamnionitis (median (IQR), 43 $(14-49)$ v $4(0-8) \mathrm{ng} / \mathrm{ml} ; \mathrm{p}<0.0024)$. 


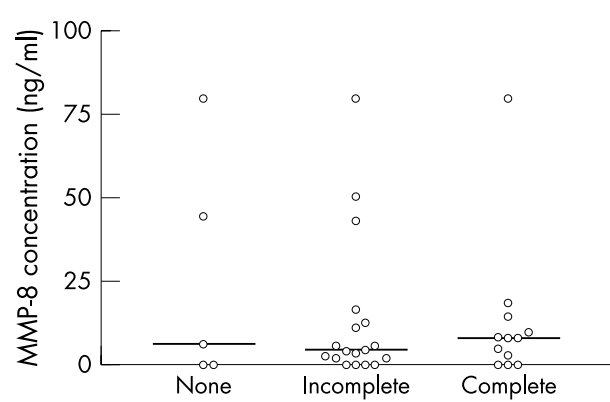

Figure 4 Matrix metalloproteinase-8 (MMP-8) concentrations according to antenatal corticosteroid administration. Median (IQR): no steroids, $6(0-54) \mathrm{ng} / \mathrm{ml}$; incomplete course, $4(2-13) \mathrm{ng} / \mathrm{ml}$; complete course, $8(2-12) \mathrm{ng} / \mathrm{ml}$.

We have shown that antenatal inflammation may upregulate lung collagenase activity and we believe that this contributes to the development of CLD. Further studies are necessary to determine true cause and effect relations for this unique inflammatory mediator. New technologies will hopefully facilitate elucidation of the biological and pathological roles of the MMPs and their inhibitors. Gene targeting and gene transfer will allow more definitive characterisation of the in vivo role of gene products. The challenge will be to control the pathological effects of these proteolytic enzymes without disrupting their vital physiological functions.

\section{Authors' affiliations}

A E Curley, D G Sweet, H L Halliday, Department of Child Health, Queen's University Belfast, and the Regional Neonatal Unit, Royal Maternity Hospital, Belfast, Northern Ireland

K J MacMahon, C M O'Connor, Department of Medicine and Therapeutics, University College Dublin, Ireland

\section{REFERENCES}

1 Yoon BH, Romero R, Park JS, et al. The relationship among inflammatory lesions of the umbilical cord (funisitis), umbilical cord plasma interleukin-6 concentration, amniotic fluid infection and neonatal sepsis. Am J Obstet Gynecol 2000; 183:1124-9.

2 Yoon BH, Romero R, Park JS, et al. Fetal exposure to an intra-amniotic inflammation and the development of cerebral palsy at three years. Am J Obstet Gynecol 2000;182:675-81.

3 Romero R, Quintero R, Nores J, Avila C, et al. Amniotic fluid white cell count: a rapid and simple test to diagnose microbial invasion of the amniotic cavity and predict preterm delivery. Am J Obstet Gynecol 1997;176:77-81.

4 Maymon E, Romero R, Pacora P, et al. Human neutrophil collagenase (matrix metalloproteinase 8 ) in parturition, premature rupture of the membranes and intrauterine infection. Am J Obstet Gynecol 2000;183:94-9.

5 Athayde N, Edwin SS, Romero R, et al. A role for matrix metalloproteinase-9 in spontaneous rupture of the fetal membranes. Am J Obstet Gynecol 1998; 179:1248-53

6 Maymon E, Romero R, Chalworapongsa T, et al. Amniotic fluid matrix metalloproteinase-8 in preterm labor with intact membranes. Am J Obstet Gynecol 2001;185:1149-55
7 Davidson JM. Biochemistry and turnover of lung interstitium. Eur Respir J 1990;3:1048-68.

8 Wright DG, Gallin Jl. Secretory responses of human neutrophils: exocytosis of specific (secondary) granules by human neutrophils during adherence in vitro and during exudation in vivo. $J$ Immunol 1979;123:258-94.

9 Murphy G, Docherty AJP. The matrix metalloproteinases and their inhibitors. Am J Respir Cell Mol Biol 1992;7:120-5

10 Sampson JE, Theve RP, Blatman RN, et al. Fetal origin of polymorphonuclear leukocytes. Am J Obstet Gynecol 1997:176:77-81.

11 Park JS, Roberto R, Yoon BH, et al. The relationship between amniotic fluid matrix metalloproteinase-8 and funisitis. Am J Obstet Gynecol 2001; 185:1156-61.

12 Sweet DG, McMahon KJ, Curley AE, et al. Type-1 collagenases in bronchoalveolar lavage fluid from preterm babies at risk of developing chronic lung disease. Arch Dis Child Fetal Neonatal Ed 2001;84:F168-71.

13 Watterberg KL, Demers LM, Scott SM, et al. Chorioamnionitis and early lung inflammation in infants in whom bronchopulmonary dysplasia develops. Pediatrics 1996:97:210-15.

14 Kotecha S, Chan B, Azam N et al. Increase in interleukin-8 and soluble intercellular adhesion molecule-1 in broncho-alveolar lavage fluid from premature infants who develop chronic lung disease. Arch Dis Child Fetal Neonatal Ed 1995;72:F90-6.

15 De Blic J, Midulla F, Barbato A, et al. Bronchoalveolar lavage in children: European Respiratory Society taskforce on bronchoalveolar lavage in children. Eur Respir J 2000;15:217-31.

16 Merritt TA, Stuard ID, Puccia J, et al. Newborn tracheal aspirate cytology: classification during respiratory distress syndrome and bronchopulmonary dysplasia. J Pediatr 1981;98:949-54.

17 Van Marter LJ, Dammann O, Allred EN, et al. Chorioamnionitis, mechanical ventilation, and postnatal sepsis as modulators of chronic lung disease in preterm infants. J Pediatr 2002;140:171-6.

18 Redline RW, Wilson-Costello D, Hack M. Placental and other perinatal risk factors for chronic lung disease in very low birth weight infants. Pediatr Res 2002;52:713-19.

19 Knauper V, Osthues A, DeClerck YA, et al. Fragmentation of human polymorphonuclear leukocyte collagenase. Biochem J 1993;291:847-54.

20 Maeda H, Okamoto T, Akaike T. Human matrix metalloprotease activation by insults of bacterial infection involving proteases and free radicals. Biol Chem 1998;379: 193-200.

21 Devaskar UP, Taylor W, Govindrajan R, et al. Hyperoxia induces interstitial (type I) and increases type IV collagenase mRNA expression and increases type I and IV collagenolytic activity in newborn rat lung. Biol Neonate type I and IV collag

22 Mallya SK, Mookhtiar KA, Gao Y, et al. Characterisation of 58-kilodalton human neutrophil collagenase: comparison with human fibroblast collagenase. Biochemistry 1990;29:10628-34.

23 Hasty KA, Hibbs MS, Kang AH, et al. Secreted forms of human neutrophil collagenase. J Biol Chem 1986;261:5645-50.

24 Finlay GA, O'Driscoll LR, Russell KJ, et al. Matrix metalloproteinase expression and production by alveolar macrophages in emphysema. Am J Respir Crit Care Med 1997;156:240-7.

25 Rannels DE, Rannels SR. Influence of the extracellular matrix on type 2 cell differentiation. Chest 1989;96:165-73.

26 Jobe AH. The new BPD: an arrest of lung development. Pediatr Res 1999:46:641-3.

27 Groneck P, Reuss D, Gotze-Speer B, et al. Effects of dexamethasone on chemotactic activity and inflammatory mediators in tracheobronchial aspirates of preterm infants at risk for chronic lung disease. J Pediatr 1993; 122:938-44.

28 Crofton PM, Shrivastava A, Wade JC, et al. Effects of dexamethasone treatment on bone and collagen turnover in preterm infants with chronic lung disease. Pediatr Res 2000;48:155-62.

29 Shapiro SD, Campbell EJ, Kobayashi DK, et al. Dexamethasone selectively modulates basal and lipopolysaccharide-induced metalloproteinase and tissue inhibitor of metalloproteinase production by human alveolar macrophages. $J$ Immunol 1991;146:2724-9.

30 Ballard PL, Granberg P, Ballard RA. Glucocorticoid levels in maternal and cord serum after prenatal betamethasone therapy to prevent respiratory distress syndrome. J Clin Invest 1975;56:1548-54. 